\title{
Generation of high-contrast, joule-level pulses based on Nd:glass chirped pulse amplification laser
}

\author{
Xiaoming $\mathrm{Lu}^{1}$, Yujie Peng ${ }^{1}$, Yanyan $\mathrm{Li}^{1}$, Xinliang Wang ${ }^{1,2}$, Xiaoyang $\mathrm{Guo}^{1}, \mathrm{Yi} \mathrm{Xu}^{1}$, and Yuxin Leng ${ }^{1}$ \\ ${ }^{1}$ State Key Laboratory of High Field Laser Physics, Shanghai Institute of Optics and Fine Mechanics, Chinese Academy of Sciences, \\ Shanghai 201800, China \\ ${ }^{2}$ School of Physics Science and Engineering, Tongji University, Shanghai 200092, China \\ (Received 1 August 2016; revised 10 September 2016; accepted 30 September 2016)
}

\begin{abstract}
We demonstrate a high-contrast, joule-level Nd:glass laser system operating at $0.5 \mathrm{~Hz}$ repetition rate based on a double chirped pulse amplification (CPA) scheme. By injecting high-contrast, high-energy seed pulses into the Nd:glass CPA stage, the pulse energy is amplified to $1.9 \mathrm{~J}$ through two optical parametric CPA stages and two Nd:glass amplifiers. The temporal contrast of compressed pulse is measured down to the level of $10^{-8}$ at tens of ps, and $10^{-10}$ near 200 ps before the main pulse, respectively.
\end{abstract}

Keywords: chirped pulse amplification; Nd:glass; temporal contrast

\section{Introduction}

Since the 1980s, chirped pulse amplification $(\mathrm{CPA})^{[1]}$ technique has been used to generate high-peak-power pulses by many laboratories and the focused intensity has reached at a level of $10^{20} \mathrm{~W} \mathrm{~cm}^{-2}$ or more. For some kinds of solid target experiments, the temporal noise intensity of a focused pulse on target should be kept below $10^{12} \mathrm{~W} \mathrm{~cm}^{-2}$ to avoid destroying the experimental conditions ${ }^{[2]}$. Then, the temporal contrast which is defined as the ratio of the intensity of the prepulses or noise background to the peak intensity of the main pulse has become a key parameter for highpeak-power laser system. The contrast enhancement methods for high-peak-power pulse have been developed. The general method is based on the high-contrast, high-energy seed-pulse injection to suppress the amplified spontaneous emission (ASE) in the amplification progress. To generate $1053 \mathrm{~nm}$ high-contrast seed pulses, many groups used optical parametric amplification (OPA) pumped by high-contrast psduration pulses which shared the same seed with the signal pulses ${ }^{[3-7]}$. With high-contrast seed injection, the contrast of the amplified pulse in the Nd:glass CPA systems had been improved, respectively ${ }^{[5-8]}$.

In the past several years, we have developed a new pulse cleaning device which combines three cascaded fs-OPAs

Correspondence to: Y. Leng, State Key Laboratory of High Field Laser Physics, Shanghai Institute of Optics and Fine Mechanics, Chinese Academy of Sciences, Shanghai 201800, China.

Email: lengyuxin@siom.ac.cn and a second harmonic generation (SHG) device to generate the high-contrast seed with sub-mJ energy ${ }^{[9,10]}$. Based on the high-contrast seed pulses, we also have demonstrated high-contrast amplified outputs at the energy level of $100 \mathrm{~mJ}^{[10,11]}$. In this paper, we further demonstrate the high-contrast output in our J-level Nd:glass laser system. This laser system mainly includes a commercial $800 \mathrm{~nm}$ Ti:sapphire $\mathrm{kHz}$ CPA laser, a pulse cleaning device which is used to generate high-contrast $1053 \mathrm{~nm}$ seed pulses, an optical parametric CPA (OPCPA) pre-amplifier, and two $1053 \mathrm{~nm} \mathrm{Nd:glass} \mathrm{amplifiers} \mathrm{in} \mathrm{which} \mathrm{the} \mathrm{pulse} \mathrm{energy} \mathrm{is}$ amplified to $1.9 \mathrm{~J}$ with $0.5 \mathrm{~Hz}$ repetition rate. The result shows that the contrast of compressed pulse is at the level of $10^{-8}$ at tens of ps and $10^{-10}$ near 200 ps before the main pulse.

\section{Laser setup}

The laser system is based on the double CPA scheme ${ }^{[12]}$ which includes a $1 \mathrm{kHz}$ Ti:sapphire CPA laser, an OPASHG temporal pulse cleaning device, an Offner stretcher, an OPCPA pre-amplifier, two Nd:glass rod amplifiers, and a Treacy compressor, as shown in Figure 1.

As shown in Figure 2, the commercial Ti:sapphire CPA laser (Spitfire, Spectra-Physics) outputs $800 \mathrm{~nm}, 40 \mathrm{fs}$, $3.3 \mathrm{~mJ}$ pulses with a repetition rate of $1 \mathrm{kHz}$. It is used as the source and pump laser of the pulse cleaning device. In the pulse cleaning device, a small part of energy of the 


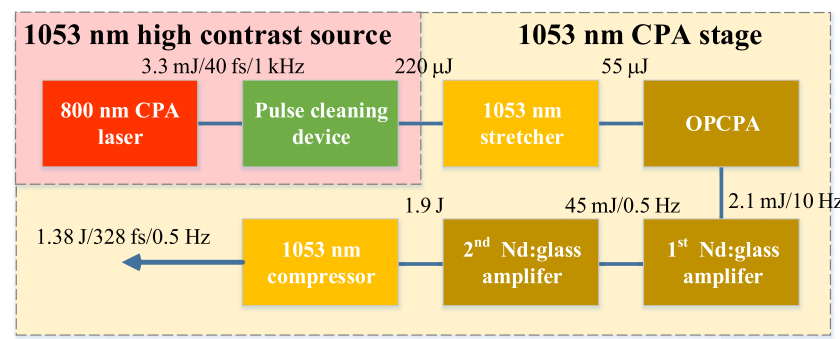

Figure 1. Schematic overview of the double CPA laser system.

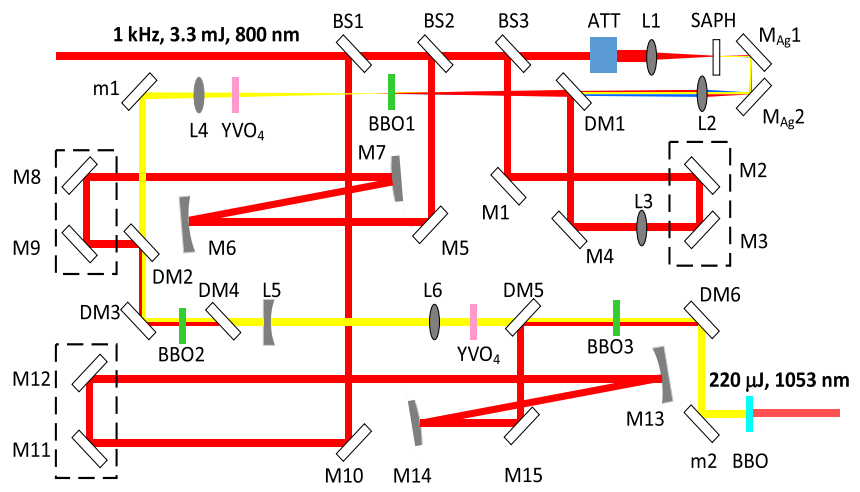

Figure 2. Layout of the OPA-SHG pulse cleaning device. BS1-BS3: beam splitter; ATT: attenuator; L1-L6: lens; SAPH: sapphire plate; $\mathrm{M}_{\mathrm{Ag}} 1, \mathrm{M}_{\mathrm{Ag}} 2$ : silver mirror; DM1-DM6: dichroic mirror; M1-M15: $800 \mathrm{~nm}$ HR mirror; $\mathrm{m} 1, \mathrm{~m} 2: 2106 \mathrm{~nm}$ HR mirror.

$800 \mathrm{~nm}$ laser is focused in a sapphire plate to generate whitelight continuum pulses. In the first OPA stage, the $1290 \mathrm{~nm}$ components of the white-light continuum pulses are used as the signals to generate $2106 \mathrm{~nm}$ idlers with the $800 \mathrm{~nm}$ pump. After two following OPA stages, the $2106 \mathrm{~nm}$ pulses are further amplified to about $450 \mu \mathrm{J}$. Finally, the pulses pass through a $\beta$-barium borate (BBO) crystal for frequency doubling to generate $220 \mu \mathrm{J}, 1053 \mathrm{~nm}$ cleaning pulses.

The spectrum is shown in Figure 3(a). Most of the energy is concentrated in a $20 \mathrm{~nm}$ bandwidth range near the gain peak of Nd:glass $(1053 \mathrm{~nm})$. The autocorrelation curve of the pulse is shown in Figure 3(b), which is corresponding to 156 fs (assuming Gaussian shape). The scanning cross correlation measurements (Sequoia-800 and Sequoia-1000, Amplitude Technologies) are shown in Figure 4. The measured contrast of the cleaned pulse is limited (about $10^{-11}$ ) by the dynamic range of the measurement device. The satellite pulse pair at $\pm 10 \mathrm{ps}$ disappears in the contrast curve of $1053 \mathrm{~nm}$ pulses, which shows the contrast is improved at least by 8 orders. There is a prepulse with intensity of about $10^{-7}$ at 51 ps before the main pulse. It may be generated by surface reflections and be amplified in OPAs.

The temporal contrast of the amplified pulse in a CPA system depends not only on the contrast of the seed pulses but also on the energy of the seed ${ }^{[13,14]}$. In the case of psOPA, limited by the energy and pulse duration of the pump
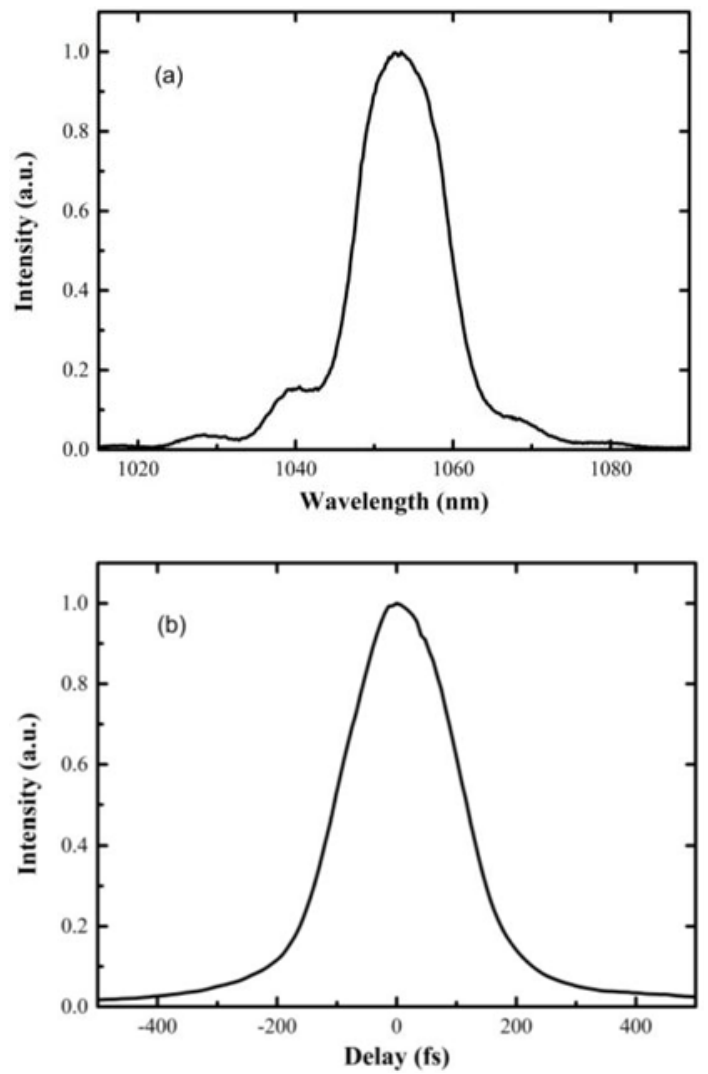

Figure 3. (a) Measured spectrum and (b) autocorrelation trace of the OPASHG cleaning pulses.

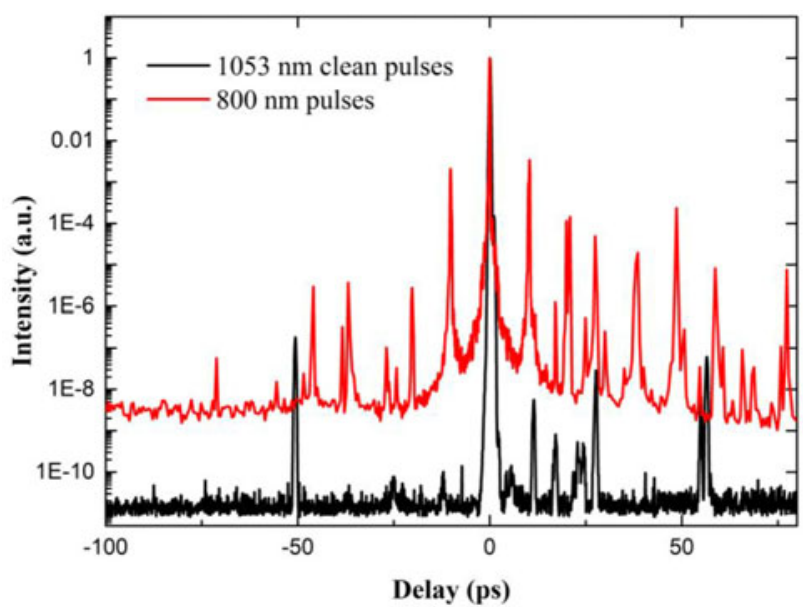

Figure 4. Third-order correlation contrast measurements of the $800 \mathrm{~nm}$ pump pulses and the $1053 \mathrm{~nm}$ OPA-SHG cleaning pulses.

pulses, the energy of the cleaned pulse is usually at the level of $\mu \mathrm{J}$ or tens of $\mu \mathrm{J}$. Compared with ps-OPAs, our fs-OPASHG cleaned pulses have two main advantages: firstly, the energy of the cleaned pulse is higher because the shorter pump duration is used which leads to higher efficiency; secondly, higher contrast could be achieved because the doubling frequency process improves the contrast further after three OPA stages ${ }^{[15]}$. 

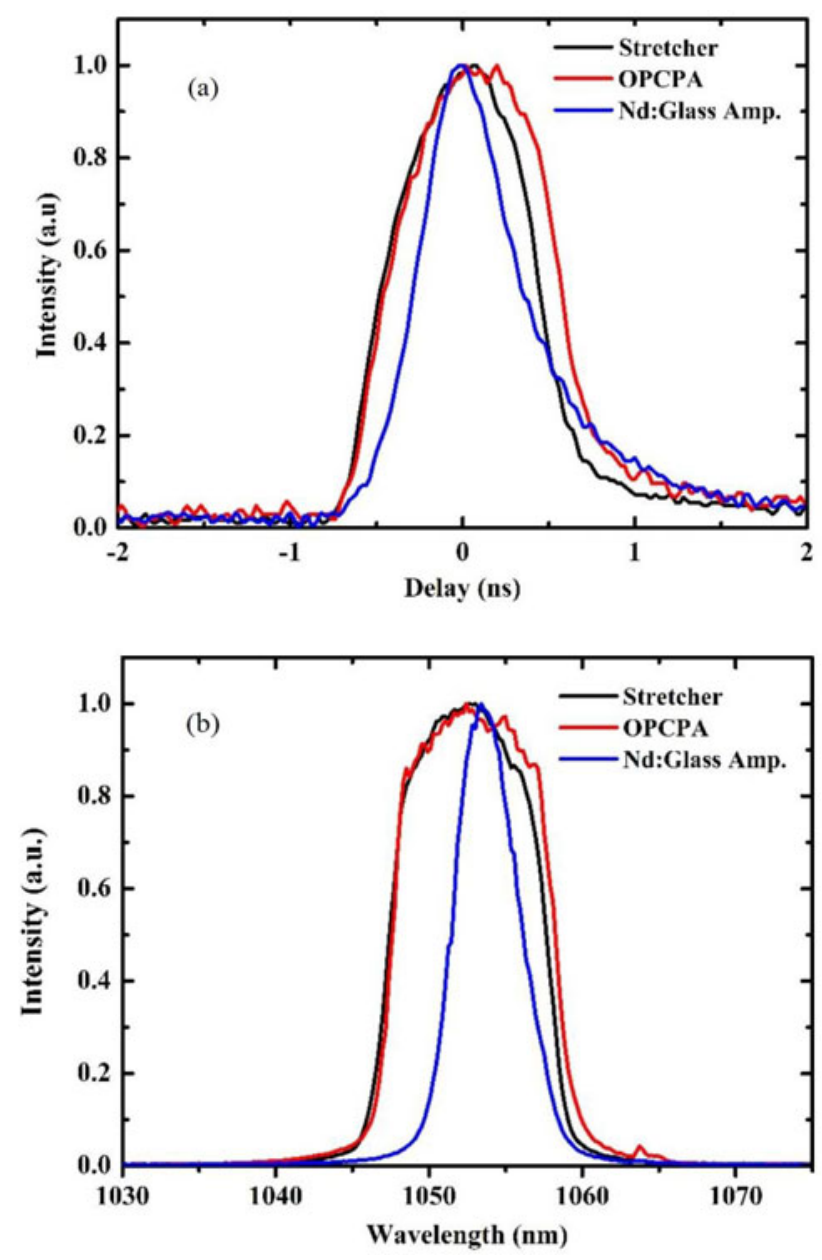

Figure 5. (a) Pulse durations; (b) spectra of the amplified pulse.

In the $1053 \mathrm{~nm}$ CPA stage, the cleaned fs pulses are stretched to about $0.95 \mathrm{~ns}$ by a two-pass Offner triplet stretcher as shown in Figure 5(a). The stretcher consists of a 1740 grooves $\mathrm{mm}^{-1}$ multilayer-dielectric grating, a concave spherical mirror $(R=-1000 \mathrm{~mm})$, and a convex spherical mirror $(R=500 \mathrm{~mm})$. The incident angle on the grating is about $71.7^{\circ}$ and the average distance between the grating and the concave mirror is about $750 \mathrm{~mm}$. A prism is inserted at the exit of the first pass to reflect the pulses back for a second pass in the stretcher. The total transmission efficiency of the stretcher is $25 \%$, indicating an output energy of $55 \mu \mathrm{J}$. The full spectrum width of the stretched pulse is about $18 \mathrm{~nm}$ as shown in Figure 5(b).

After the stretcher, the laser pulse is amplified in a twostage OPCPA pre-amplifier ${ }^{[10]}$. The OPCPA pre-amplifier includes a 20-mm-thick BBO crystal in the first OPCPA stage and an 8-mm-thick BBO crystal in the second OPCPA stage. Both of them are cut at $22.85^{\circ}$ for type I phase matching. The two OPCPA stages are pumped in a cascade way by a single longitudinal mode $Q$-switched Nd:YAG laser (Quanta-Ray lab-150, Spectra-Physics) which can generate

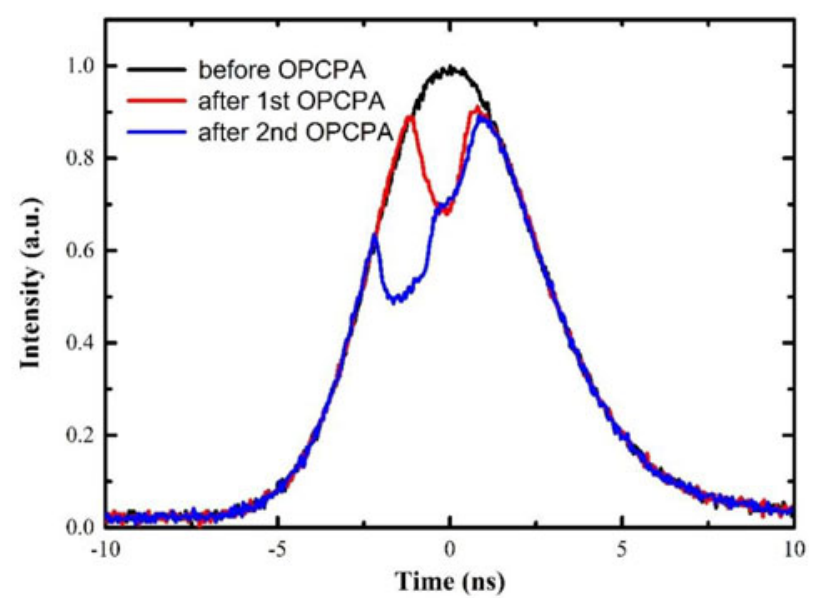

Figure 6. Time profiles of the pump pulse in the OPCPA pre-amplifier.

$5.5 \mathrm{~ns}, 532 \mathrm{~nm}$ pulses with a repetition of $10 \mathrm{~Hz}$. In the first OPCPA stage, the signal pulse is overlapped by the pump pulse completely while there is $1.5 \mathrm{~ns}$ additional time delay between the pump pulse and the signal pulse considering the second OPCPA stage. The energy of the pump laser is adjusted to about $170 \mathrm{~mJ}$. The top-hat pump beam is relay imaged and down-collimated to $4 \mathrm{~mm}$ on the first OPCPA crystal. The $1053 \mathrm{~nm}$ signal pulse is amplified to about $15 \mathrm{~mJ}$ corresponding to a total gain of 300 in the two-stage OPCPA amplifier.

Both the characters of the amplified pulse (Figure 5) and the depletion of pump energy (Figure 6) indicate that the OPCPA is operating in a nonsaturation state. By blocking or opening the seed pulses of the OPCPA, we can measure that the intensity ratio between the parametric fluorescence and the amplified pulse is about $3 \times 10^{-7}$ using a photodiode and a set of calibrated neutral density filters. Multiplying by a compression factor of about $2 \times 10^{-4}$, the relative intensity of the parametric fluorescence after pulse compression is about $6 \times 10^{-11}$. Considering the suppression of the signal injection, the real intensity of the parametric fluorescence will be lower.

A nonsaturated OPCPA is beneficial for high-contrast amplification ${ }^{[16]}$, but it is adverse to obtain the homogeneous beam. As shown in Figure 7(c), the beam profile of the OPCPA amplified pulse is seriously affected by the poor pump beam profile sensitively. To improve the homogeneity of the signal pulse beam profile, the signal pulse beam is expanded by a Galileo expander, and then a $\phi 4 \mathrm{~mm}$ diameter signal pulse beam with good profile is picked out from the expanded beam by a serrated-tooth apodizer. After filtering off the high-frequency components, the signal pulse beam is homogenized as shown in Figure 7(d), and $2.1 \mathrm{~mJ}$ of energy is remained.

After the OPCPA pre-amplifier, the pulse is amplified by following two Nd:glass amplifiers, which are upgraded from the previous $100-\mathrm{mJ}$-level amplifier ${ }^{[10]}$. As shown in 

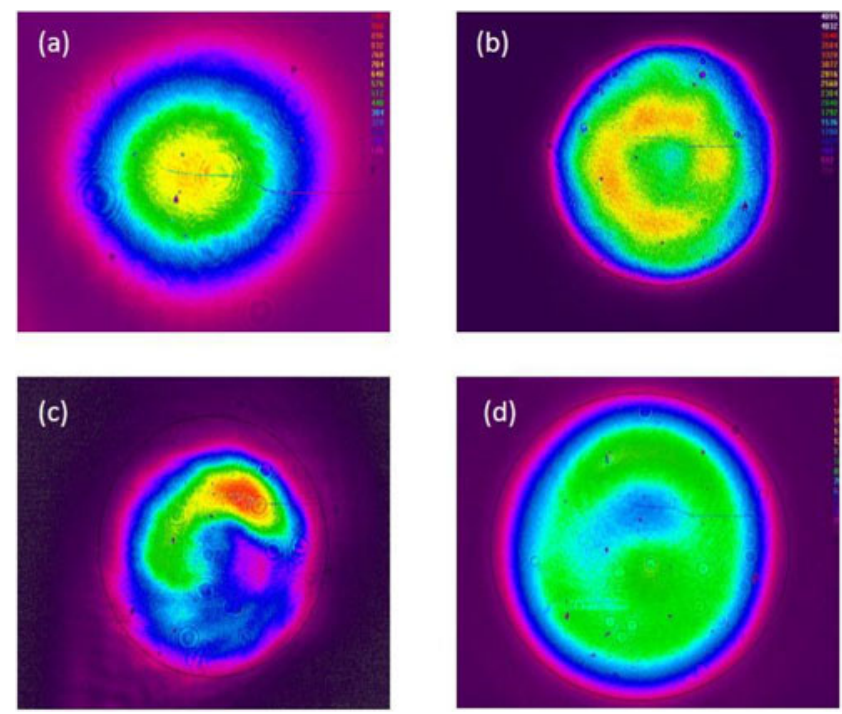

Figure 7. Beam profiles in the OPCPA pre-amplifier: (a) seed beam profile; (b) pump beam profile; (c) OPCPA amplified beam profile; (d) picked-out homogenized beam profile.

Figure 8, the first amplifier consists of a quarter wave plate, two $\phi 7 \mathrm{~mm}$ single-flashlamp-pumped Nd:glass rod modules, a $90^{\circ}$ quartz rotator, a Faraday Rotator, a thin film polarizer, a 1:1 telescope system. The telescope makes the principle planes of the two rods image each other. The imaging system and the $90^{\circ}$ quartz rotator exchange the tangential polarization states of the two rods to compensate thermalinduced birefringence. A pinhole filter is set on the focus plane to filter off the high-frequency components of the beam. The quarter wave plate is inserted before the first glass module to generate the circular polarization for reducing the B-integral in the amplifier. The pulse is amplified twice in this amplifier stage, leading to an output of $45 \mathrm{~mJ}$. The repetition rate is set at $0.5 \mathrm{~Hz}$.

Before injected into the following $\phi 12 \mathrm{~mm} \mathrm{Nd:glass} \mathrm{rod}$ amplifier, the laser beam is expanded to $\phi 10 \mathrm{~mm}$. The second amplifier has the similar structure as the first one, and the imaging system is set in a vacuum environment to

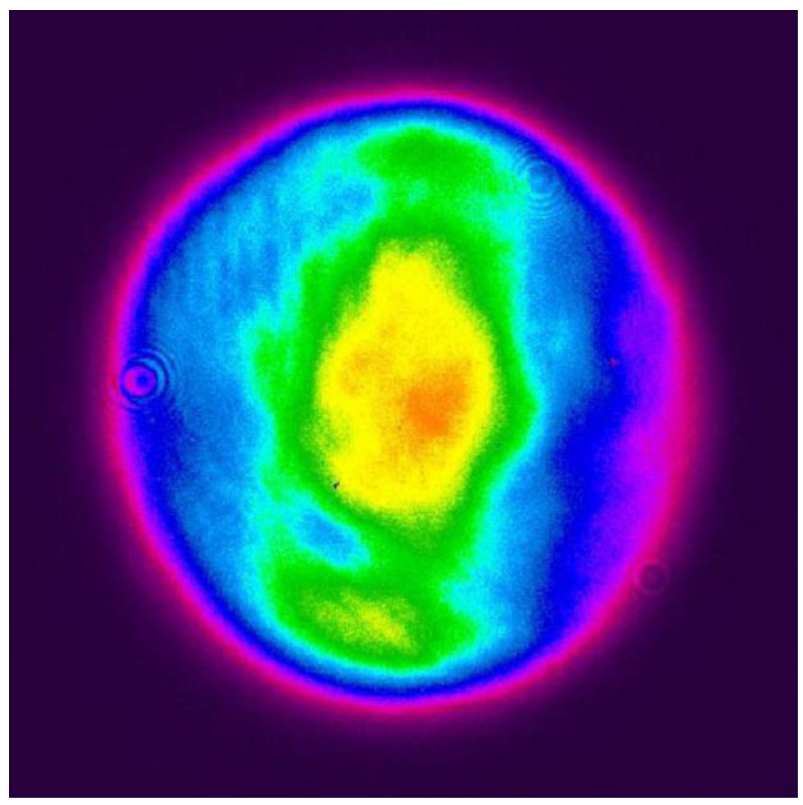

Figure 9. Near-field beam profile of the amplified pulse.

avoid laser-induced breakdown in the focus plane (Figure 8). The pulse energy is boosted to $1.9 \mathrm{~J}$ at the repetition rate of $0.5 \mathrm{~Hz}$. The total gain of the two $\mathrm{Nd}$ :glass rod amplification stages is about 900 .

The spectra and the time profiles of the amplified pulse are shown in Figure 5. Because of the gain narrowing effect in the Nd:glass amplifiers, the spectrum of the amplified pulse reduces to about $4.7 \mathrm{~nm}$ (FWHM), and the duration also reduces to about $0.65 \mathrm{~ns}$ (FWHM).

The near-field beam profile of the amplified pulse is shown in Figure 9. The strong area in the beam profile limits further energy improvement of the amplified pulse. This strong area in the vertical direction is mainly caused by the single-flashlamp-pumped rod modules in the first Nd:glass rod amplifier. The uniform pumped modules will be used to replace the present modules in the future.

The sampling pulse of the amplified pulse is sent to the compressor. The compressor consisted of two

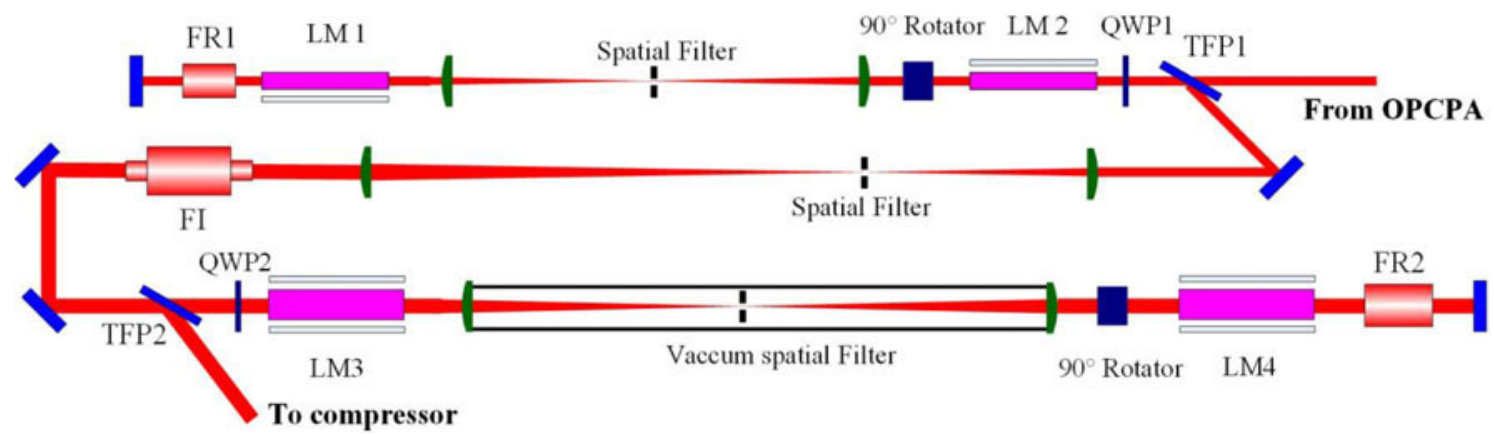

Figure 8. Layout of the two Nd:glass rod amplifiers. TFP1, TFP2: thin film polarizer; QWP1, QWP2: quarter wave plate; LM1, LM2: single-flashlamppumped Nd:glass rod module; FR1, FR2: Faraday Rotator; FI: Faraday Isolator; LM3, LM4: double-flashlamp-pumped Nd:glass rod module. 


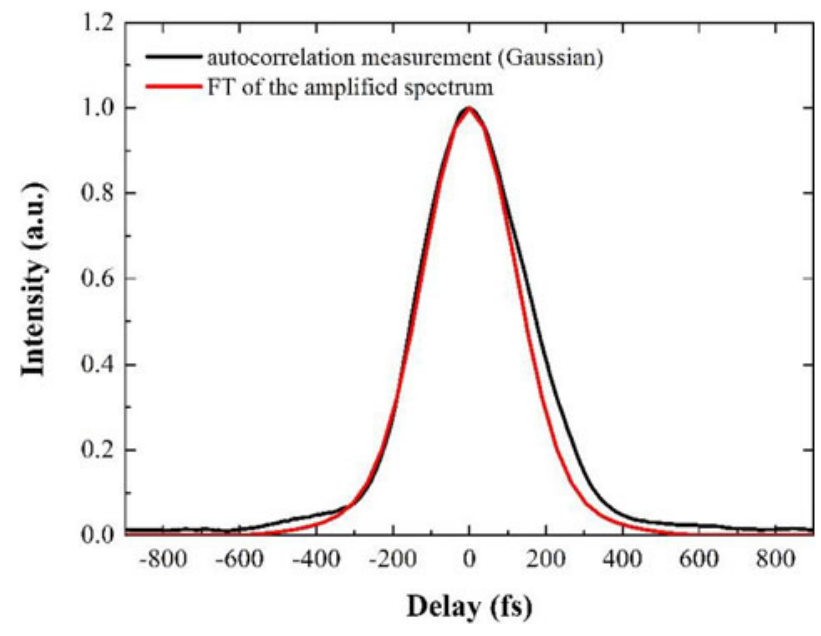

Figure 10. Autocorrelation measurement and Fourier-transform-limited pulse duration.

1740 grooves $\mathrm{mm}^{-1}$ golden gratings and a roof mirror. The autocorrelation measurement shows that the duration of the compressed pulse is about 328 fs (Figure 10, assuming Gaussian shape), while the Fourier transform limit of the amplified pulse (Figure 5) is $295 \mathrm{fs}$.

\section{Contrast measurement}

The third-order correlation scanning contrast measurement of the compressed pulse is shown in Figure 11. Limited by the low operating repetition and the low long-time stability of the laser system, we choose a scanning step of 2 ps except the range near the main pulse In the measurement, every point is averaged by 4 times. Therefore, it takes about one hour to complete a contrast measurement. Here, the prepulse at 51 ps before the main pulse is missed due to 2-ps step and mixed in the noise (about $10^{-8}$ ).

It shows that the contrast is about $10^{-8}$ in the tens of ps range before the main pulse. The measurement also shows that a slow rising slope at the level of $10^{-10}$ starts from about 200 ps before the main pulse. The ASE noise is the main contrast degradation origin for most CPA lasers in which the pulses from oscillators are used as the seeds directly. Here, as the seed pulses for the Nd:glass laser system are high contrast and high energy, ASE noise can be suppressed efficiently. As shown in our previous report ${ }^{[10]}$, the contrast pedestal comes from the stretching-compressing process mainly. The theoretical analysis of Dorrer et al. indicated the high-frequency spectral phase noise in stretcher or compressor could impact the contrast ${ }^{[17]}$. It was also indicated that the convex mirror in Offner triplet stretcher has an important impact on the contrast degradation ${ }^{[18]}$. In the future, we will optimize our stretcher design to improve the contrast further.

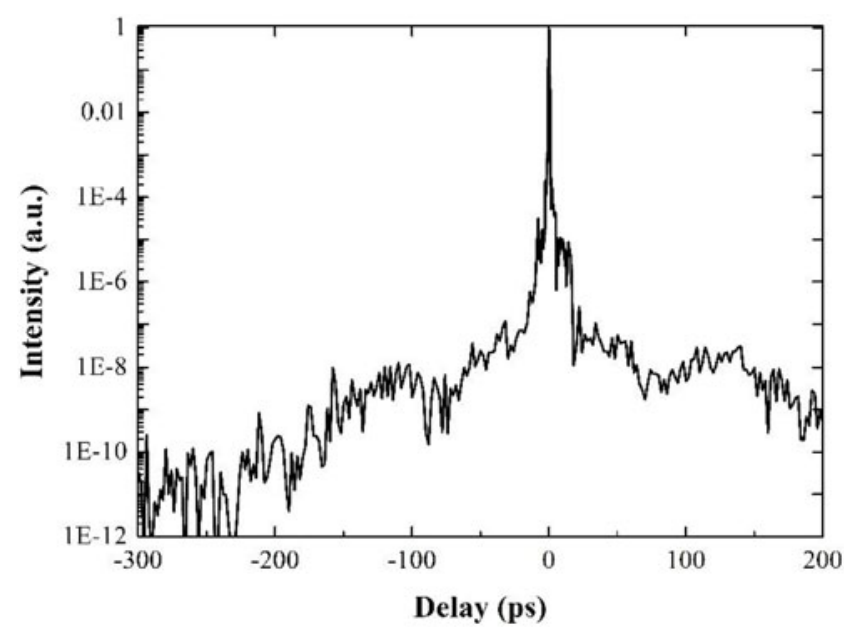

Figure 11. Third-order correlation contrast measurement of the compressed pulse.

\section{Conclusion}

In conclusion, by injecting high-contrast, high-energy seed pulses into the Nd:glass CPA stage, the pulse energy is amplified to $1.9 \mathrm{~J}$ through two OPCPA stages and two $\mathrm{Nd}$ :glass amplifiers. The temporal contrast measurement shows was measured down to the level of $10^{-8}$ at tens of ps, and $10^{-10}$ near 200 ps before the main pulse, respectively. The higher-power Nd:glass laser system with repetition rate and high contrast is being developed.

\section{References}

1. D. Strickland and G. Mourou, Opt. Commun. 56, 219 (1985).

2. D. Umstadter, Phys. Plasmas 8, 1774 (2001).

3. C. Dorrer, I. Begishev, A. Okishev, and J. Zuegel, Opt. Lett. 32, 2143 (2007).

4. R. C. Shah, R. P. Johnson, T. Shimada, K. A. Flippo, J. C. Fernandez, and B. M. Hegelich, Opt. Lett. 34, 2273 (2009).

5. I. Musgrave, W. Shaikh, M. Galimberti, A. Boyle, C. Hernandez-Gomez, K. Lancaster, and R. Heathcote, Appl. Opt. 49, 6558 (2010).

6. D. I. Hillier, S. Elsmere, M. Girling, N. Hopps, D. Hussey, S. Parker, P. Treadwell, D. Winter, and T. Bett, Appl. Opt. 53, 6938 (2014).

7. F. Wagner, C. Joao, J. Fils, T. Gottschall, J. Hein, J. Körner, J. Limpert, M. Roth, T. Stöhlker, and V. Bagnoud, Appl. Phys. B 116, 429 (2014).

8. C. Dorrer, A. Consentino, D. Irwin, J. Qiao, and J. Zuegel, J. Opt. 17, 094007 (2015).

9. Y. Li, Y. Huang, J. Wang, Y. Xu, X. Lu, D. Wang, Y. Leng, R. Li, and Z. Xu, Laser Phys. Lett. 10, 075403 (2013).

10. X. Lu, Y. Peng, Y. Li, X. Guo, Y. Leng, Z. Sui, Y. Xu, and X. Wang, Chin. Opt. Lett. 14, 023201 (2016).

11. X. M. Lu, Y. X. Leng, Z. Sui, Y. Y. Li, Z. X. Zhang, Y. Xu, X. Y. Guo, and Y. Q. Liu, Laser Phys. 24, 105301 (2014).

12. M. Kalashnikov, E. Risse, H. Schönnagel, and W. Sandner, Optics Lett. 30, 923 (2005).

13. V. V. Ivanov, A. Maksimchuk, and G. Mourou, Appl. Opt. 42, 7231 (2003). 
14. V. Bagnoud, J. Zuegel, N. Forget, and C. Le Blanc, Opt. Express 15, 5504 (2007).

15. Y. Huang, C. Zhang, Y. Xu, D. Li, Y. Leng, R. Li, and Z. Xu, Opt. Lett. 36, 781 (2011).

16. H. Kiriyama, M. Mori, Y. Nakai, T. Shimomura, M. Tanoue,
A. Akutsu, H. Okada, T. Motomura, S. Kondo, and S. Kanazawa, Opt. Commun. 282, 625 (2009).

17. C. Dorrer and J. Bromage, Opt. Express 16, 3058 (2008).

18. J. Bromage, C. Dorrer, and R. Jungquist, JOSA B 29, 1125 (2012). 